\title{
The Neuroprotective Mechanism of Low-Frequency rTMS on Nigral Dopaminergic Neurons of Parkinson's Disease Model Mice
}

\author{
Qiaoyun Dong, ${ }^{1}$ Yanyong Wang, ${ }^{2}$ Ping Gu, ${ }^{2}$ Rusheng Shao, ${ }^{1}$ Li Zhao, \\ Xiqi Liu, ${ }^{1}$ Zhanqiang Wang, ${ }^{3}$ and Mingwei Wang ${ }^{2}$ \\ ${ }^{1}$ Fifth Department of Neurology, Cangzhou Central Hospital, No. 16 Xinhua Western Road, Cangzhou, Hebei 061000, China \\ ${ }^{2}$ Hebei Province Key Laboratory of Brain Aging and Cognitive Neuroscience, No. 16 Xinhua Western Road, \\ Cangzhou, Hebei 061000, China \\ ${ }^{3}$ Department of Neurology, Cangzhou Central Hospital, No. 16 Xinhua Western Road, Cangzhou, Hebei 061000, China
}

Correspondence should be addressed to Mingwei Wang; wmw@jyyy.com.cn

Received 27 October 2014; Revised 24 January 2015; Accepted 24 January 2015

Academic Editor: Antonio Pisani

Copyright (C) 2015 Qiaoyun Dong et al. This is an open access article distributed under the Creative Commons Attribution License, which permits unrestricted use, distribution, and reproduction in any medium, provided the original work is properly cited.

Background. Parkinson's disease is a neurodegenerative disease in elder people, pathophysiologic basis of which is the severe deficiency of dopamine in the striatum. The purpose of the present study was to evaluate the neuroprotective effect of lowfrequency rTMS on Parkinson's disease in model mice. Methods. The effects of low-frequency rTMS on the motor function, cortex excitability, neurochemistry, and neurohistopathology of MPTP-induced Parkinson's disease mice were investigated through behavioral detection, electrophysiologic technique, high performance liquid chromatography-electrochemical detection, immunohistochemical staining, and western blot. Results. Low-frequency rTMS could improve the motor coordination impairment of Parkinson's disease mice: the resting motor threshold significantly decreased in the Parkinson's disease mice; the degeneration of nigral dopaminergic neuron and the expression of tyrosine hydroxylase were significantly improved by low-frequency rTMS; moreover, the expressions of brain derived neurotrophic factor and glial cell line derived neurotrophic factor were also improved by low-frequency rTMS. Conclusions. Low-frequency rTMS had a neuroprotective effect on the nigral dopaminergic neuron which might be due to the improved expressions of brain derived neurotrophic factor and glial cell line-derived neurotrophic factor. The present study provided a theoretical basis for the application of low-frequency rTMS in the clinical treatment and recovery of Parkinson's disease.

\section{Introduction}

Parkinson's disease (PD) is a major neurodegenerative disease in elderly population, the symptom of which includes bradykinesia, resting tremor, muscular rigidity, and gait disturbance $[1,2]$. The onset and development of PD is found to be closely related to the cell loss and eosinophilic intracytoplasmic aggregation in substantia nigra (SN) [3]. Recent studies have reported a significant decrease of the striatal dopamine (DA) in PD patients [4] and the conclusion was supported by that most degenerated cells in $\mathrm{SN}$ expressed proteins involved in the synthesis, degeneration, and transport of DA $[5,6]$. The underlying mechanism of this neuron loss in PD patients remains unrevealed; however, hypothesis that the degeneration of nigral dopaminergic neuron (NDN) is linked to a lack of trophic support was proposed. Those trophic factors include brain derived neurotrophic factor (BDNF) and glial cell line derived neurotrophic factor (GDNF), which have been reported to be downregulated in the SN from PD patients. Therapeutic methods focusing on increasing the level of BDNF and GDNF in the SNare are being developed.

Despite the fact that the nigral DA cell loss has been taken as a specific characteristic of PD, the etiology of PD is still not well understood, which makes it difficult to develop perfect therapeutic strategy. Traditional therapies such as DA 
precursor and operation can only contribute to the control of the disorder but fail to prevent neuronal degeneration along with lots of side effects [7, 8]. Development of promising alternative therapeutic strategies is imperative.

In recent studies, it was suggested that repetitive transcranial magnetic stimulation (rTMS) might be a potential treatment for different neuropsychiatric diseases [9-13]. The technique is a novel noninvasive and painless treatment associated with few or mild side effects which can produce lasting changes in excitability as well as activity in cortical regions and functionally connected cortical or subcortical regions [913]. Several studies have showed that high-frequency $(>1 \mathrm{~Hz})$ and low-frequency $(<1 \mathrm{~Hz})$ rTMS both had a beneficial effect on motor functions in PD patients [11, 12]; moreover, lowfrequency rTMS had a higher safety in clinical practice than high-frequency rTMS. Rationales of rTMS on PD involve the increasing release of DA and BDNF in certain brain areas [14]. However, the mechanism through which rTMS exerts therapeutic effect stays unclear.

In the present study, we investigated the effects of lowfrequency rTMS on motor function, cortex excitability, neurochemistry, neurohistopathology of 1-methyl-4-phenyl1,2,3,6-tetrahydropyridine- (MPTP-) induced PD mice by behavioral detection, electrophysiologic technique, high performance liquid chromatography-electrochemical detection (HPLC-ECD), immunohistochemical staining, and western blot. We hoped that our study would reveal the mechanism underlying the effect of low-frequency rTMS on PD and improve the practical application of low-frequency rTMS in treating $\mathrm{PD}$ patients in the future.

\section{Materials and Methods}

2.1. PD Model Establishment and Low-Frequency rTMS Treatment. Fifty-six eight-week-old male C57BL/6j mice weighing 240-260 g were randomly divided into four groups, 14 mice for each group: NS group (control group, healthy mice receiving normal saline group injection), PD group (acute PD model group, mice receiving MPTP injection to establish acute PD model), s-rTMS group (sham group, PD model mice exposed to the noise during the rTMS stimulation), and rTMS group (low-frequency rTMS treatment group, PD mice receiving low-frequency rTMS stimulation). For PD model establishment, the mice received four MPTP injections ( $15 \mathrm{mg} / \mathrm{kg}$, s.c., dissolved in $0.9 \%$ saline) with twohour intervals at the 1st day of the experiment to establish an acute PD model while the mice in NS group were injected with the same volume of saline instead of MPTP at the same time points. All animal experiments were conducted in the accordance with the Institutional Animal Ethics Committee and Animal Care Guidelines of Hebei Medical University that governed the use of experimental animals.

Twenty-four hours after the last injection, the mice in rTMS group received five trains of $1 \mathrm{~Hz}$ stimulation for $25 \mathrm{~s}$ at the intensity of 1 Tesla with a $10 \mathrm{~mm}$ diameter circular coil. The interval between each strain was $2 \mathrm{~min}$. Centers of the coils were $15 \mathrm{~mm}$ higher than mice heads. During the stimulation, all the animals were awakened. The treatment was conducted one time per day for mice in rTMS group and lasted for 14 consecutive days at the same time point each day. Mice in s-rTMS group were exposed to the same noise during the rTMS simulation for 14 days with center of coils being more than $10 \mathrm{~cm}$ apart from the mice heads. No treatment was performed on mice in NS and PD groups.

2.2. Effect of Low-Frequency rTMS on the Behavior of PD Model Mice. The mice were assessed for the motor movement using an automated locomotor activity test and a rotarod test before agents injections and at 1st, $3 \mathrm{rd}, 7 \mathrm{th}$, and 14 th day after the last injection. For automated locomotor activity test, the observation was conducted using a ZH-YLS-ZA Automated Locomotor Activity Control Instrument (Huaibeizhenghua Biological Instrument, Co., LTD) according to the user introduction. Rotary test was conducted based on the method of Vijitruth et al. [15]: mice were placed in a $20 \mathrm{~cm}$ diameter rotating bar, and the number of the rotation before mice left the bar was recorded. Each mouse was tested for five times at each time point and the interval between each time was $5 \mathrm{~min}$.

\subsection{The Effect of Low-Frequency rTMS on the Cortex Excitabil-} ity of PD Model Mice. Thirty-two eight-week-old male C57BL/6j mice weighing 240-260 g were randomly divided into four experimental groups as described above, 8 mice for each group. Treatments were performed as described above. Then mice were assessed for their resting motor threshold (RMT) according to previous studies [16, 17] before agents injection and at 1st, 3rd, 7th, and 14th day after the last injection: mice were anesthetized with $40 \mathrm{mg} / \mathrm{kg}$ chloralhydrate and stimulated using Magpro X100 Magnetic Stimulator (Dantec Dynamics A/S, Denmark) by placing coil at left brain of the mice. RMT values of the hind leg gastrocnemius were recorded by collecting the magnetic stimulation signal using Counterpoint Electromyography (Oxford Instruments, UK).

2.4. Effect of Low-Frequency rTMS on the Level of DA and Its Metabolites in the Striatum of PD Model Mice. Twentyfour male C57BL/6J mice weighing 240-260 g were randomly divided into four experimental groups as described above, 6 mice for each group. Treatments were performed on each group as described above. $24 \mathrm{~h}$ after the last stimulation of low-frequency rTMS, mice in each group were executed by breaking the necks, and the level of DA, homovanillic acid (HVA), and 3,4-dihydroxyphenylacetic acid (DOPAC) in mice striatum was detected using high performance liquid chromatography-electrochemical detection (HPLCECD) following standard procedures in Beijing Institute of Neurosciences, Capital Medical University.

2.5. Effect of Low-Frequency rTMS on the Function of NDN of PD Model Mice. Fifty-six male C57BL/6J mice weighing 240$260 \mathrm{~g}$ were randomly divided into four experimental groups as described above, 14 mice for each group. Treatments were performed on each group as described above. $24 \mathrm{~h}$ after the last stimulation of low-frequency rTMS, eight mice from each group were anesthetized with $40 \mathrm{mg} / \mathrm{kg}$ chloralhydrate, perfused with $30 \mathrm{~mL}$ normal saline and $100 \mathrm{~mL} 40 \mathrm{~g} / \mathrm{L}$ paraformaldehyde solution, and brain samples containing SN were then quickly frozen and cut into sections for immunohistochemical staining of tyrosine hydroxylase (TH), BDNF, 
and GDNF proteins in the SN: samples from each group were fixed with $3 \%$ hydrogen peroxide and blocked with $10 \%$ normal goat serum for $60 \mathrm{~min}$. They were then incubated separately with primary rabbit antibodies against $\mathrm{TH}$ (1:5000, Chemicon), BDNF (1:100 ABcam), and GDNF $(1: 15, \mathrm{ABcam})$ at $4^{\circ} \mathrm{C}$ overnight. Samples were rewarmed for $60 \mathrm{~min}$ at $37^{\circ} \mathrm{C}$ before incubating with goat anti-rabbit IgG $\left(1: 100\right.$, Neomarker) for another $60 \mathrm{~min}$ at $37^{\circ} \mathrm{C}$. Then samples were incubated with horseradish peroxidase (HRP, 1:100) for $60 \mathrm{~min}$ at $37^{\circ} \mathrm{C}$ and then with $\mathrm{DAB}-\mathrm{H}_{2} \mathrm{O}_{2}$ for 5 min to develop color. Observation of the immunohistochemical staining was conducted using a microscope under 100x magnification. Corrected optical density (COD) value was calculated as the difference between OD values of immunoreactive cells and those of pyramidal tracts cells.

Fresh tissue was dissected from the SN of midbrain of the left six mice in each group after cervical dislocation. Total protein was extracted for TH, BDNF, and GDNF detection by western blot following standard procedures: the extracts were boiled with loading buffer for five minutes and then subject to sodium dodecyl sulfate polyacrylamide gel electrophoresis (SDS-PAGE) on 10\% gels. Targeted proteins were transferred onto polyvinylidene difluoride sheets. The membranes were washed with TBST for three times, $20 \mathrm{~min}$ for each time. Then the membranes were incubated with primary antibody (rabbit antibody against TH (1:1000, Chemicon); rabbit antibody against $\mathrm{BDNF}(1: 500$, Chemicon); rabbit antibody against GDNF (1:25, ABcam); rabbit antibody against $\beta$ actin $(1: 300, A B c a m))$ overnight at room temperature. After additional three washes, secondary antibody (goat anti-rabbit $\operatorname{IgG}(1: 1000$, Neomarker)) was added and the membrane was incubated for four hours. After three final washes, the blots were developed using Beyo ECL Plus reagent and the results were detected in the Gel Imaging System. The content of the targeted protein was expressed as relative optical densities (RODs), which were calculated as the ratio of OD values of the targeted proteins to that of $\beta$-actin detected by Quantity One software (Bio-Rad Laboratories, Inc.).

2.6. Statistical Analysis. All the data were expressed in the form of mean \pm SD. Multiple comparisons were conducted by $q$ method and correlation was calculated using Pearson coefficiency. All the statistical analyses were conducted using SPSS version 16.0 (IBM, Armonk, NY, USA) with a significant level being 0.05 .

\section{Results}

3.1. Low-Frequency rTMS Treatment Improved the Behavior of PD Model Mice. Shortly after the first injection of MPTP, model mice showed performance of piloerection, bradykinesia, stroub tail reaction, instability of gait, tremor, and the toes of the hind feet widely separated. Those performances remained in MPTP-treated mice for 5 to $6 \mathrm{~h}$. Similar symptoms were observed after the subsequent injections, and the symptoms of bradykinesia, stiffness, and instability of gait strengthened with the injection of MPTP. However, all the mice showed recovery as regards external appearance $24 \mathrm{~h}$ after injection. No abnormality was observed in the

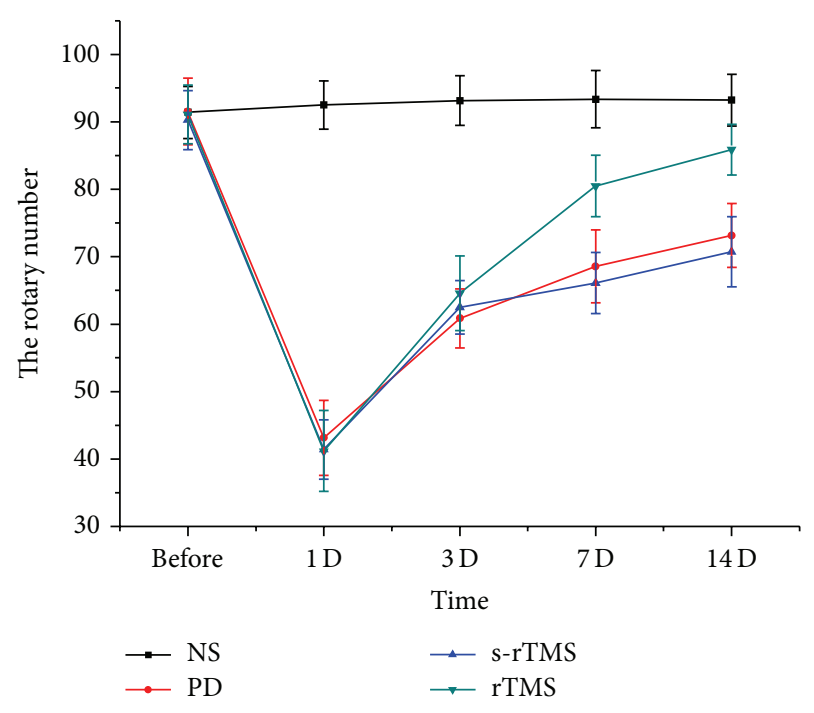

FIGURE 1: The comparison of rotarod testing in different groups at different time points.

control group. No dysfunction was observed in the stimulated animals during or after low-frequency rTMS. The results of automated locomotor activity test showed that treatment of low-frequency rTMS had no improvement on the locomotor activity of model mice (Figure 1; See Supplementary Table S1 in Supplementary Material available online at http://dx.doi.org/10.1155/2015/564095); however, significant effect of low-frequency rTMS on the improvement of rotary number was observed in rotary test (Figure 2; Table S2).

3.2. Low-Frequency rTMS Treatment Improved the Cortex Excitability of PD Model Mice. Significant decreases of the RMT of mice were observed in MPTP-treated groups compared to that of the NS group after the first injection of MPTP (Figure 3; Table S3) $(P<0.05)$. The decrease persisted for PD and s-rTMS groups for the left injections but with a mild pattern. For rTMS group, increase of the RMT of mice was observed since the 3rd day after the last injection. The difference of RMT between rTMS group and PD/s-rTMS groups was significant at the 7th day and 14th day (Figure 3; Table S3) $(P<0.05)$.

3.3. Low-Frequency rTMS Increased the Level of DA and Related Metabolites in the Striatum of PD Model Mice. A dramatic decline of DA level was observed in the striatum of PD mice (Figure 4; Table S4) $(P<0.05)$. DOPAC and HVA level also decreased compared to NS group (Figure 4; Table S4) $(P<0.05)$. After low-frequency rTMS treatment, the DA, DOPAC, and HVA levels in rTMS group were significantly increased compared with PD and s-TMS groups (Figure 4; Table S4) $(P<0.05)$. No significant difference was found between s-rTMS and PD group (Figure 4; Table S4).

3.4. The Protective Effect of Low-Frequency rTMS on the NDN of PD Model Mice. The results of immunohistochemical staining were shown in Figure 5 and Table S5. Generally, the 


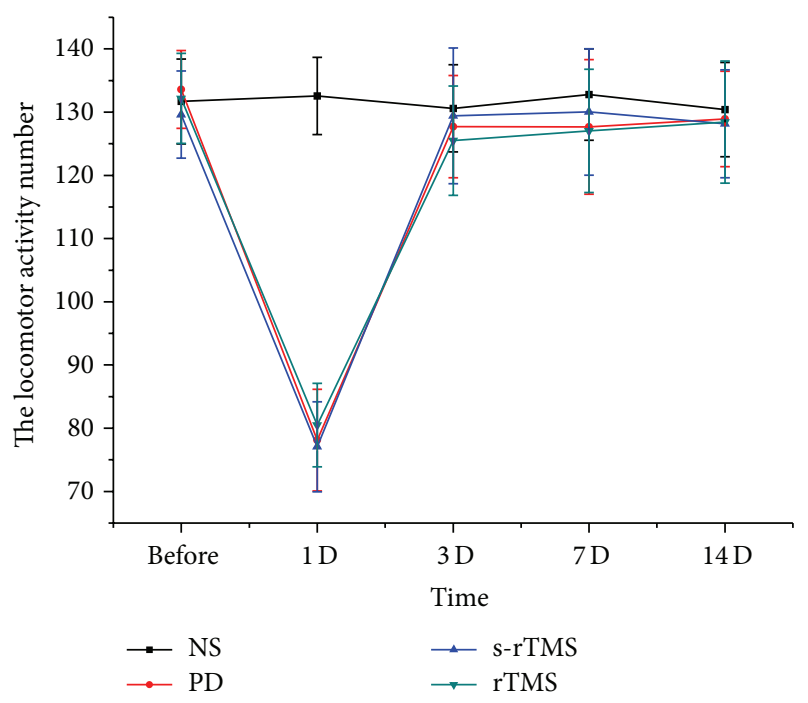

FIgURE 2: The comparison of the locomotor activity in different groups at different time points.

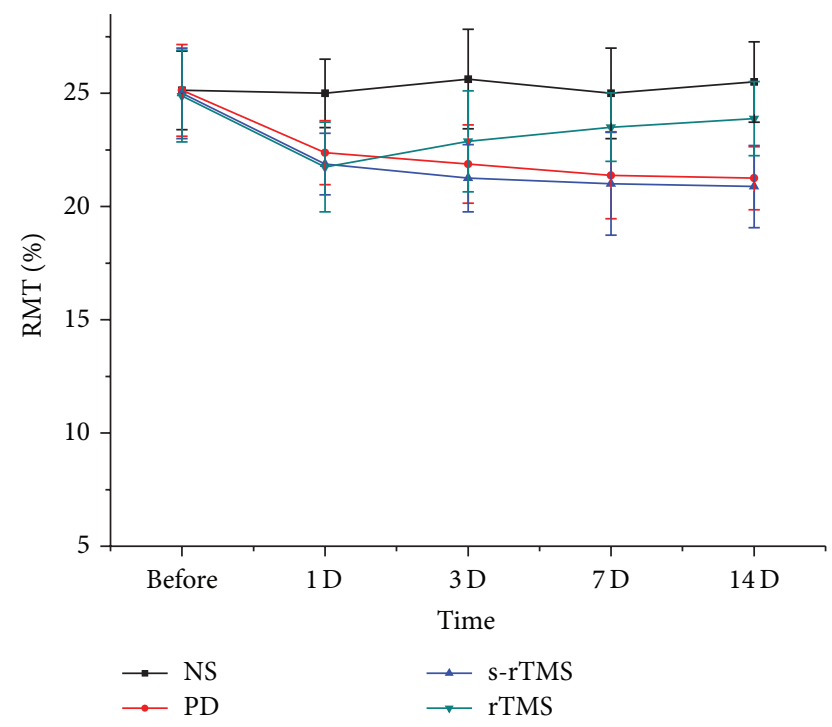

FIGURE 3: The comparison of RMT of mice in different groups at different time points.

expressions of TH, BDNF (including two bands: monomer and homodimer), and GDNF all significantly declined in PD model mice. However, the symptoms were reversed after treatment of low-frequency rTMS. And the results showed that the changes of COD of $\mathrm{TH}$ were positively related to those of BDNF and GDNF (Table 1). Same patterns were also observed in the western blot of TH, BDNF, and GDNF, which confirmed the protective effect of low-frequency rTMS on the NDN of PD model mice (Figure 6; Tables 2 and S6).

\section{Discussions}

PD is thought to be a result of complex rearrangements in the neuronal circuits which is responsible for motor activity

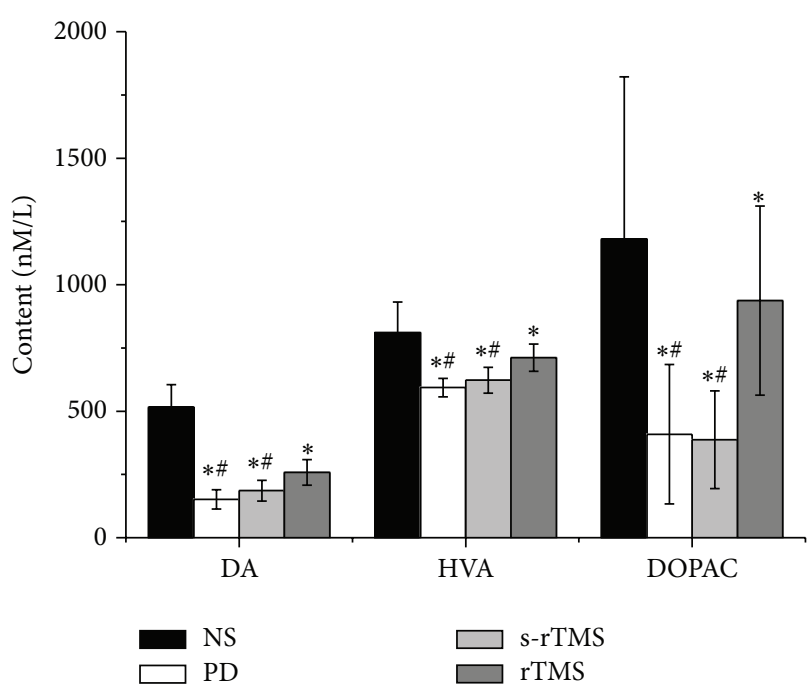

FIGURE 4: The effects of low-frequency rTMS on the content of DA and its metabolites in the striatum of PD model mice. ${ }^{*}$ Significantly different from NS group, $P<0.05$; " significantly different from rTMS group, $P<0.05$.

TABLE 1: Correlation analysis between COD values of TH and BDNF or GDNF as illustrated by immunohistochemical staining. in SN of mice.

\begin{tabular}{lccc}
\hline & Groups & $\begin{array}{c}\text { Correlation } \\
\text { Coefficient }\end{array}$ & $P$ value \\
\hline \multirow{3}{*}{ TH and BDNF } & NS & 0.906 & 0.002 \\
& PD & 0.894 & 0.003 \\
& s-rTMS & 0.903 & 0.002 \\
TH and GDNF & rTMS & 0.967 & 0.000 \\
& NS & 0.959 & 0.000 \\
& PD & 0.898 & 0.002 \\
& rTTMS & 0.730 & 0.040 \\
\hline
\end{tabular}

[18-20]. The study of Fox et al. [21] firstly described the application of rTMS in treating PD, which reported that subthreshold, high-frequency $(5 \mathrm{~Hz})$ rTMS over M1 induced a dramatic improvement of reaction and movements times, as well as the performance on the grooved pegboard test. These benefits were confirmed by other groups [22-24]. In the present, we focused on the therapeutic effect of lowfrequency $r$ TMS on $\mathrm{PD}$ for its higher safety in clinical practice compared to high-frequency rTMS.

Although, in the behavior test, locomotor activity improvement of model mice was not related to low-frequency rTMS treatment, significant improvement of rotary number was observed in the rTMS group (Figures 1 and 2). Such results should suggest that low-frequency rTMS acts on a subclinical level and is strongly effective in normalizing endophenotypic abnormalities of the MPTP induced PD models but has not yet the sufficient efficacy to fully restore 
NS

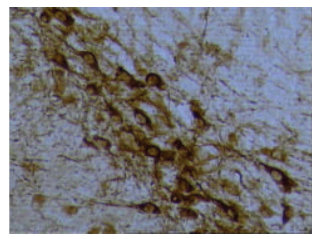

s-rTMS

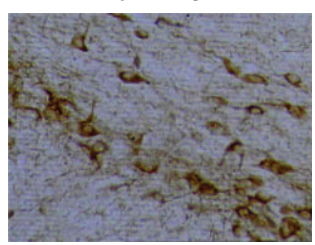

NS

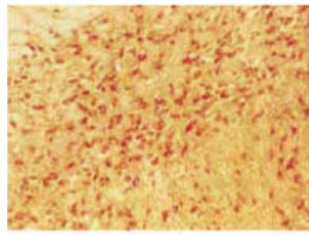

s-rTMS

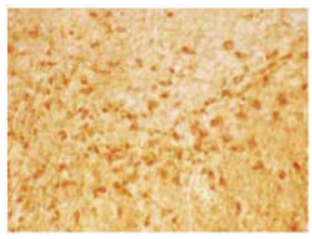

NS

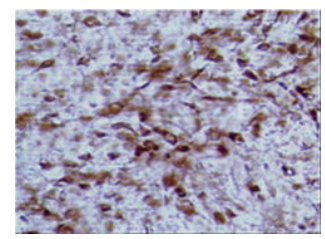

s-rTMS

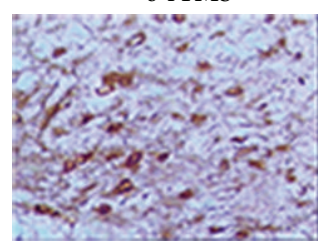

$\mathrm{PD}$

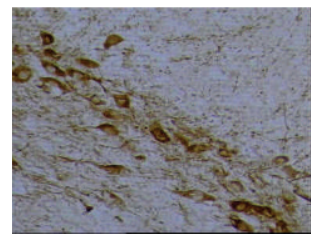

rTMS

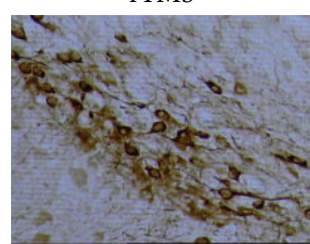

PD

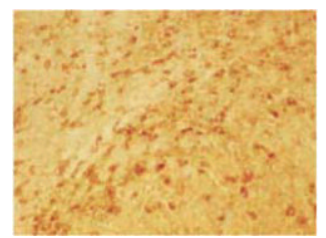

rTMS

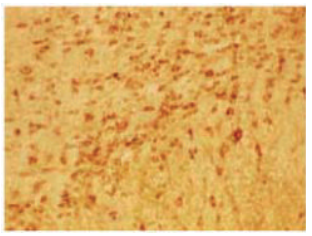

PD

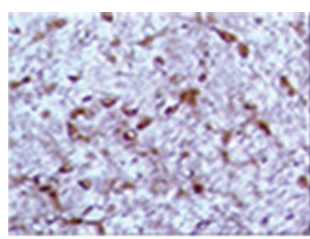

rTMS

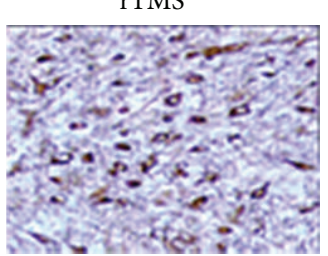

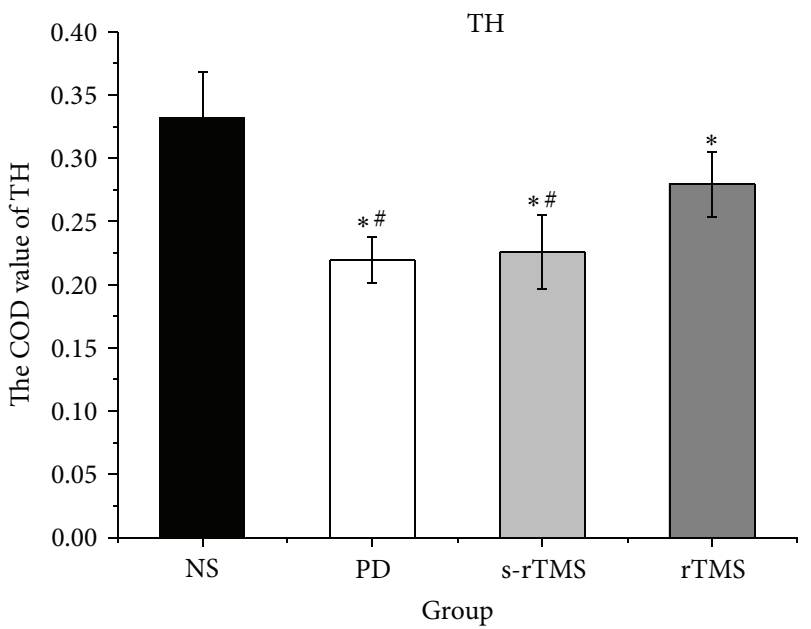

BDNF

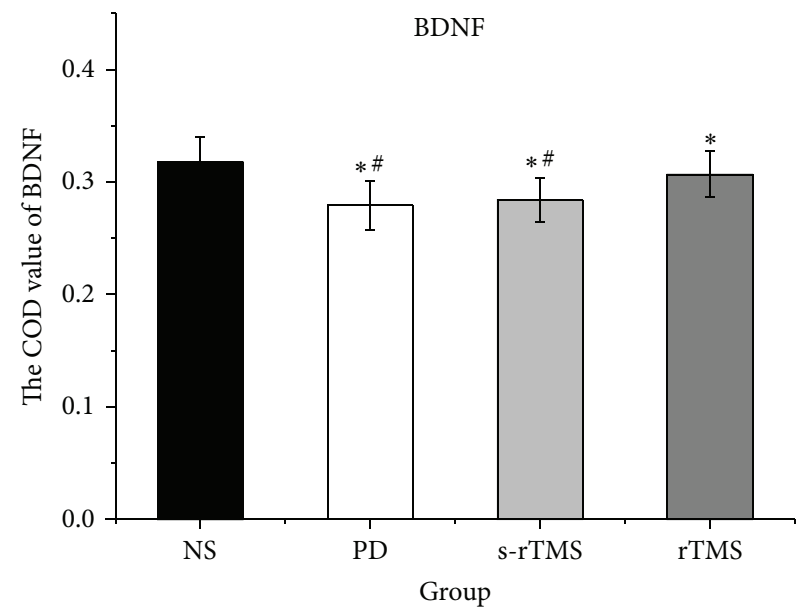

GDNF

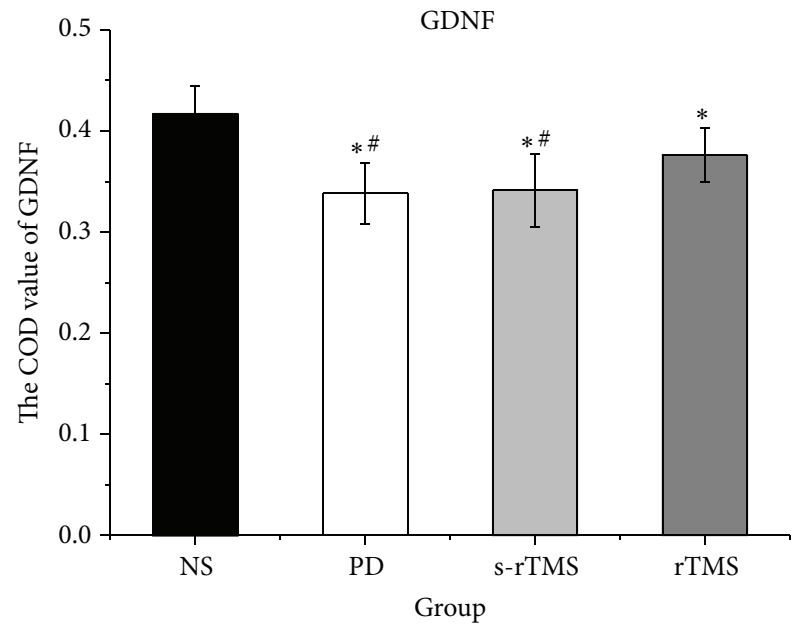

FIGURE 5: The effect of low-frequency rTMS on the expression of TH, BDNF, and GDNF as illustrated by immunohistochemical staining.

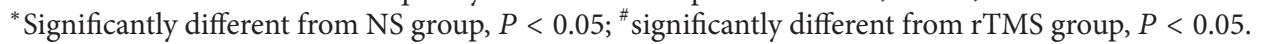

even behavior impairment. In addition, the effect of lowfrequency rTMS on rotary number increased with the time of treatment, showing the accumulative effect of therapy. Lowfrequency rTMS could also act on the cortex excitability of PD model mice and increased of the RMT of model mice since the 3rd day after the last MPTP injection. The difference of RMT between rTMS group and PD/s-rTMS groups was significant at the 7th day and 14th day (Figure 3). Previous studies showed that high-frequency rTMS could increase the excitability of motor cortex [25], while rTMS lower than $1 \mathrm{~Hz}$ could significantly reduce the excitability of cortex with a longer effect [26]. Kinds of brain circuits in PD patients were 

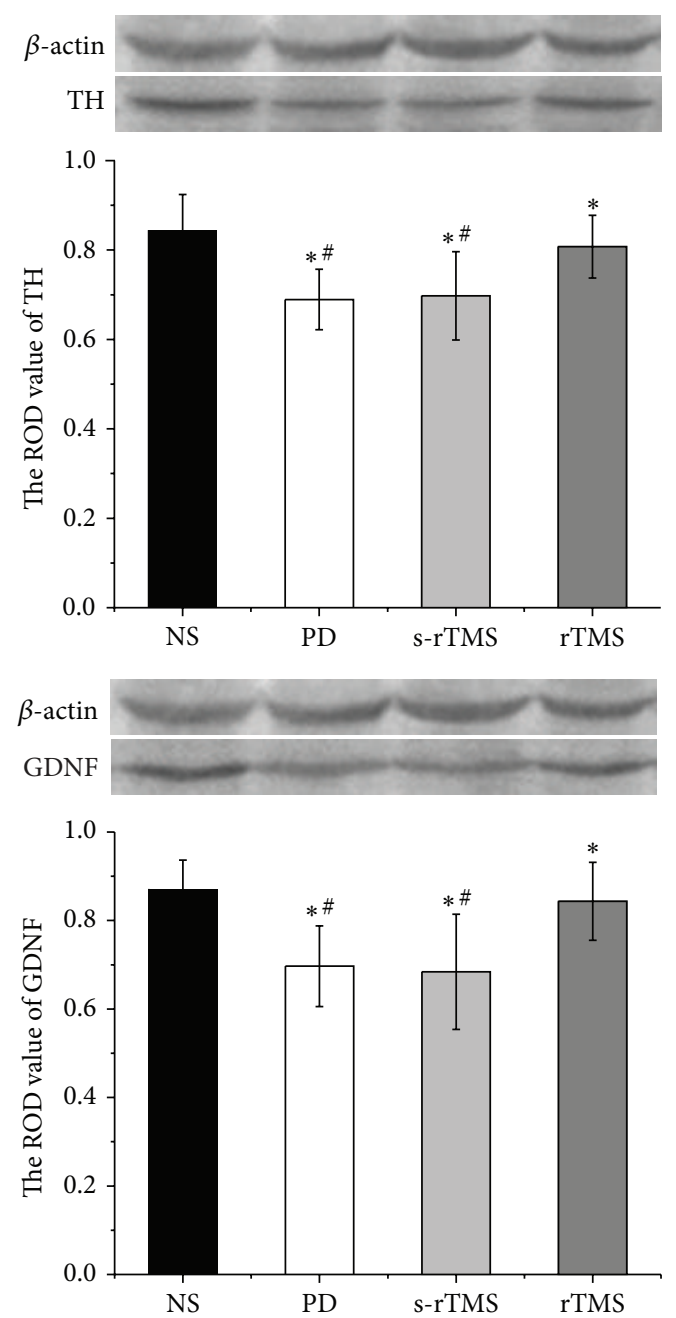

$\beta$-actin

BDNF-homodimer

BDNF-monomer

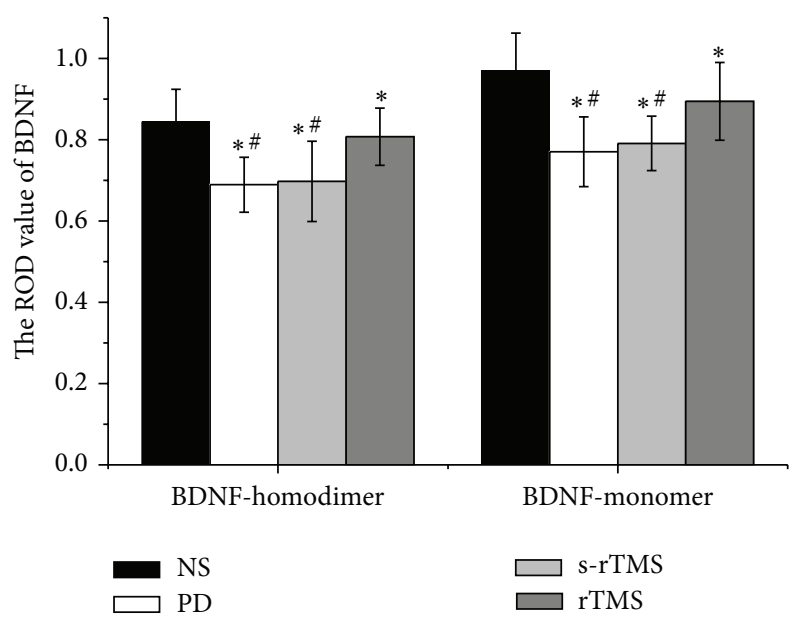

FIGURE 6: The effect of low-frequency rTMS on the expression of TH, BDNF, and GDNF as illustrated by western blot. ${ }^{*}$ Significantly different from NS group, $P<0.05$; ${ }^{\#}$ significantly different from rTMS group, $P<0.05$.

blocked resulting from the inefficient synthesis of DA. By changing the local excitability and activity of neuron, lowfrequency rTMS could influence the long distant cortex and subcortical area and reverse the abnormality in different brain circuits. In addition, the effect of low-frequency rTMS on RMT also had an accumulative effect (Figure 3), which did not show significant difference from those of PD and s-rTMS group until the 7 th day of the treatment.

The injection of MPTP could significantly decline the synthesis of DA and its metabolites in the PD model mice (Figure 4). Most of DA distributed in SN striate system, and the main metabolites of DA were HVA and DOPAC. Those molecules all play important roles in motion control [27] and content of DA in basal ganglia declining to $30 \%$ of normal level would cause clinical symptoms of PD [28]. Compared with PD and s-rTMS groups, the syntheses of DA, HVA, and DOPAC were much higher in rTMS group (Figure 4).
Previous study has also indicated that effect of rTMS function is not only through the activation of cortex of PD but also by influencing basal ganglia through striatum [29]. This conclusion was consistent with our results, in which the levels of TH, BDNF, and GDNF were all strengthened in rTMS group (Figures 5 and 6). TH has been taken as the marker enzyme of neurons producing dopamine in brain [30]. The increase of TH would directly improve the synthesis of DA.

Recent studies have proposed that the degeneration of SN may be due to a lack of neurotrophic factor [31], and the influence of rTMS on the expression of BDNF and GDNF could lead to the protective effect of NDN. BDNF has a critical role in cell differentiation, neuronal survival, migration, dendritic arborization, synaptogenesis, and synaptic plasticity [32]. GDNF promotes the growth, regeneration, and survival of $\mathrm{SN}$ dopamine neurons. Both of the two neurotrophins have been reported to decease in PD [33-36]. Results of immunohistochemical staining and 
TABLE 2: Correlation analysis between ROD values of TH and BDNF/GDNF as illustrated by western blot.

\begin{tabular}{lccc}
\hline & Groups & $\begin{array}{c}\text { Correlation } \\
\text { Coefficient }\end{array}$ & $P$ value \\
\hline \multirow{2}{*}{ TH and BDNF- } & NS & 0.887 & 0.018 \\
monomer & PD & 0.872 & 0.024 \\
& s-rTMS & 0.858 & 0.029 \\
& rTMS & 0.820 & 0.046 \\
\hline \multirow{3}{*}{ TH and BDNF- } & NS & 0.828 & 0.042 \\
homodimor & PD & 0.898 & 0.015 \\
& s-rTMS & 0.842 & 0.036 \\
& rTMS & 0.839 & 0.037 \\
\hline \multirow{3}{*}{ TH and GDNF } & NS & 0.889 & 0.018 \\
& PD & 0.845 & 0.034 \\
& s-rTMS & 0.908 & 0.012 \\
& rTMS & 0.824 & 0.044 \\
\hline
\end{tabular}

western blot showed that the content of BDNF and GDNF dramatically declined in model mice, but the expressions were all improved significantly after a consecutive treatment of low-frequency rTMS (Figures 5 and 6).

Although our study has provided some evidence for the effect of low-frequency rTMS on the protection of NDN, other reports have also revealed the same effect of highfrequency rTMS treatment. In a study of Lee et al. [37], it was reported that chronic $10 \mathrm{~Hz}$ rTMS enhanced the expression of BDNF, GDNF, and increased DA positive neurons in rat model of PD. Based on the results of both studies, it seemed that the protective effect of rTMS might not be due to the frequency. Given the higher safety of low-frequency rTMS, we recommended that application of low-frequency rTMS was the first option in clinical treatment of neurodegenerative disease such as PD.

\section{Conclusions}

In conclusion, our results showed that low-frequency rTMS had a considerable effect on the physiological characteristics of MPTP induced PD model mice and could be a potential therapy of $\mathrm{PD}$. The mechanism might be due to the neuroprotective effect on NDN by the improved expressions of BDNF and GDNF. And we suggested that further studies on lowfrequency rTMS in treating PD should be conducted. Systematical evaluation of the relevant methodological features would facilitate the establishment of more prominent and longer lasting effects of the therapy.

\section{Abbreviation}

PD: Parkinson's disease

SN: Substantia nigra

DA: Dopamine

NDN: Nigral dopaminergic neuron

BDNF: Brain derived neurotrophic factor
GDNF: Glial cell line-derived neurotrophic factor

rTMS: Repetitive transcranial magnetic stimulation

COD: Corrected optical density

ROD: Relative optical density.

\section{Conflict of Interests}

The authors declare that they have no competing interests.

\section{Authors' Contribution}

Qiaoyun Dong made the major contribution to the experiment and draft writing; Yanyong Wang, Ping Gu, Rusheng Shao, Li Zhao, and Xiqi Liu conducted the immunohistochemical staining and western blot; Zhanqiang Wang and Mingwei Wang revised the draft and approved the final submission.

\section{Acknowledgment}

This work was supported by the National Natural Science Foundation of Hebei Province, China (C2007000842).

\section{References}

[1] J. A. Driver, G. Logroscino, J. M. Gaziano, and T. Kurth, "Incidence and remaining lifetime risk of Parkinson disease in advanced age," Neurology, vol. 72, no. 5, pp. 432-438, 2009.

[2] A. S. Buchman, J. M. Shulman, S. Nag et al., "Nigral pathology and parkinsonian signs in elders without Parkinson disease," Annals of Neurology, vol. 71, no. 2, pp. 258-266, 2012.

[3] J. G. Greenfield and F. D. Bosanquet, "The brain-stem lesions in Parkinsonism," Journal of Neurology Neurosurgery Psychiatry, vol. 16, no. 4, pp. 213-226, 1953.

[4] O. Hornykiewicz, "Dopamine (3-hydroxytyramine) and brain function," Pharmacological Reviews, vol. 18, no. 2, pp. 925-964, 1966.

[5] K. Lloyd and O. Hornykiewicz, "Parkinson's disease: activity of L-dopa decarboxylase in discrete brain regions," Science, vol. 170, no. 3963, pp. 1212-1213, 1970.

[6] A. Kastner, E. C. Hirsch, M. T. Herrero, F. Javoy-Agid, and Y. Agid, "Immunocytochemical quantification of tyrosine hydroxylase at a cellular level in the mesencephalon of control subjects and patients with Parkinson's and Alzheimer's disease," Journal of Neurochemistry, vol. 61, no. 3, pp. 1024-1034, 1993.

[7] J. E. Ahlskog, "Slowing Parkinson's disease progression: recent dopamine agonist trials," Neurology, vol. 60, no. 3, pp. 381-389, 2003.

[8] O. Arias-Carrión, N. Freundlieb, W. H. Oertel, and G. U. Höglinger, "Adult neurogenesis and Parkinson's disease," CNS \& Neurological Disorders-Drug Targets, vol. 6, no. 5, pp. 326-335, 2007.

[9] V. Walsh and A. Cowey, "Transcranial magnetic stimulation and cognitive neuroscience," Nature Reviews: Neuroscience, vol. 1, no. 1, pp. 73-79, 2000.

[10] M. C. Ridding and J. C. Rothwell, "Is there a future for therapeutic use of transcranial magnetic stimulation?" Nature Reviews Neuroscience, vol. 8, no. 7, pp. 559-567, 2007. 
[11] F. Fregni and A. Pascual-Leone, "Technology insight: noninvasive brain stimulation in neurology-perspectives on the therapeutic potential of rTMS and tDCS," Nature Clinical Practice Neurology, vol. 3, no. 7, pp. 383-393, 2007.

[12] J. Málly and T. W. Stone, "New advances in the rehabilitation of CNS diseases applying rTMS," Expert Review of Neurotherapeutics, vol. 7, no. 2, pp. 165-177, 2007.

[13] A. Post and M. E. Keck, "Transcranial magnetic stimulation as a therapeutic tool in psychiatry: what do we know about the neurobiological mechanisms?" Journal of Psychiatric Research, vol. 35, no. 4, pp. 193-215, 2001.

[14] C. C. Real, A. F. B. Ferreira, G. P. Chaves-Kirsten, A. S. Torrão, R. S. Pires, and L. R. G. Britto, "BDNF receptor blockade hinders the beneficial effects of exercise in a rat model of Parkinson's disease," Neuroscience, vol. 237, pp. 118-129, 2013.

[15] R. Vijitruth, M. Liu, D.-Y. Choi, X. V. Nguyen, R. L. Hunter, and G. Bing, "Cyclooxygenase-2 mediates microglial activation and secondary dopaminergic cell death in the mouse MPTP model of Parkinson's disease," Journal of Neuroinflammation, vol. 3, article 6, 2006.

[16] A. Mori, S. Ohashi, M. Nakai, T. Moriizumi, and Y. Mitsumoto, "Neural mechanisms underlying motor dysfunction as detected by the tail suspension test in MPTP-treated C57BL/6 mice," Neuroscience Research, vol. 51, no. 3, pp. 265-274, 2005.

[17] E. Rousselet, C. Joubert, J. Callebert et al., "Behavioral changes are not directly related to striatal monoamine levels, number of nigral neurons, or dose of parkinsonian toxin MPTP in mice," Neurobiology of Disease, vol. 14, no. 2, pp. 218-228, 2003.

[18] T. Boraud, E. Bezard, D. Guehl, B. Bioulac, and C. Gross, "Effects of L-DOPA on neuronal activity of the globus pallidus externalis (GPe) and globus pallidus internalis (GPi) in the MPTP-treated monkey," Brain Research, vol. 787, no. 1, pp. 157-160, 1998.

[19] M. R. DeLong, "Primate models of movement disorders of basal ganglia origin," Trends in Neurosciences, vol. 13, no. 7, pp. 281285, 1990.

[20] M. Filion and L. Tremblay, "Abnormal spontaneous activity of globus pallidus neurons in monkeys with MPTP-induced parkinsonism," Brain Research, vol. 547, no. 1, pp. 142-151, 1991.

[21] P. Fox, R. Ingham, M. S. George et al., "Imaging human intracerebral connectivity by PET during TMS," NeuroReport, vol. 8, no. 12, pp. 2787-2791, 1997.

[22] T. Fukudome, H. Goto, H. Izumoto, H. Matsuo, and N. Shibuya, "The effects of repetitive transcranial magnetic stimulation (rTMS) in the patients with Parkinson's disease," Rinshō Shinkeigaku, vol. 42, no. 1, pp. 35-37, 2002.

[23] E. M. Khedr, J. C. Rothwell, O. A. Shawky, M. A. Ahmed, and A. Hamdy, "Effect of daily repetitive transcranial magnetic stimulation on motor performance in Parkinson's disease," Movement Disorders, vol. 21, no. 12, pp. 2201-2205, 2006.

[24] M. P. Lomarev, S. Kanchana, W. Bara-Jimenez, M. Iyer, E. M. Wassermann, and M. Hallett, "Placebo-controlled study of rTMS for the treatment of Parkinson's disease," Movement Disorders, vol. 21, no. 3, pp. 325-331, 2006.

[25] F. Maeda, J. P. Keenan, J. M. Tormos, H. Topka, and A. PascualLeone, "Interindividual variability of the modulatory effects of repetitive transcranial magnetic stimulation on cortical excitability," Experimental Brain Research, vol. 133, no. 4, pp. 425-430, 2000.

[26] E. M. Wassermann, J. Grafman, C. Berry et al., "Use and safety of a new repetitive transcranial magnetic stimulator," Electroencephalography and Clinical Neurophysiology, vol. 101, no. 5, pp. 412-417, 1996.
[27] M. E. Keck, T. Welt, M. B. Müller et al., "Repetitive transcranial magnetic stimulation increases the release of dopamine in the mesolimbic and mesostriatal system," Neuropharmacology, vol. 43, no. 1, pp. 101-109, 2002.

[28] M. Sedelis, K. Hofele, G. W. Auburger, S. Morgan, J. P. Huston, and R. K. W. Schwarting, "MPTP susceptibility in the mouse: behavioral, neurochemical, and histological analysis of gender and strain differences," Behavior Genetics, vol. 30, no. 3, pp. 171$182,2000$.

[29] G. E. Alexander and M. D. Crutcher, "Functional architecture of basal ganglia circuits: neural substrates of parallel processing," Trends in Neurosciences, vol. 13, no. 7, pp. 266-271, 1990.

[30] S. C. Daubner, J. Melendez, and P. F. Fitzpatrick, "Reversing the substrate specificities of phenylalanine and tyrosine hydroxylase: aspartate 425 of tyrosine hydroxylase is essential for LDOPA formation," Biochemistry, vol. 39, no. 32, pp. 9652-9661, 2000.

[31] M. B. H. Youdim, W. Maruyama, and M. Naoi, "Neuropharmacological, neuroprotective and amyloid precursor processing properties of selective MAO-B inhibitor antiparkinsonian drug, rasagiline," Drugs of Today, vol. 41, no. 6, pp. 369-391, 2005.

[32] C. W. Cotman and N. C. Berchtold, "Exercise: a behavioral intervention to enhance brain health and plasticity," Trends in Neurosciences, vol. 25, no. 6, pp. 295-301, 2002.

[33] N. Tajiri, T. Yasuhara, T. Shingo et al., "Exercise exerts neuroprotective effects on Parkinson's disease model of rats," Brain Research, vol. 1310, pp. 200-207, 2010.

[34] Y.-S. Lau, G. Patki, K. Das-Panja, W.-D. Le, and S. O. Ahmad, "Neuroprotective effects and mechanisms of exercise in a chronic mouse model of Parkinson's disease with moderate neurodegeneration," European Journal of Neuroscience, vol. 33, no. 7, pp. 1264-1274, 2011.

[35] D. M. Gash, Z. Zhang, and G. Gerhardt, "Neuroprotective and neurorestorative properties of GDNF," Annals of Neurology, vol. 44, no. 3, pp. S121-S125, 1998.

[36] R. Grondin and D. M. Gash, "Glial cell line-derived neurotrophic factor (GDNF): a drug candidate for the treatment of Parkinson's disease," Journal of Neurology, vol. 245, no. 3, pp. P35-P42, 1998.

[37] J. Y. Lee, S. H. Kim, A.-R. Ko et al., "Therapeutic effects of repetitive transcranial magnetic stimulation in an animal model of Parkinson's disease," Brain Research, vol. 1537, pp. 290-302, 2013. 


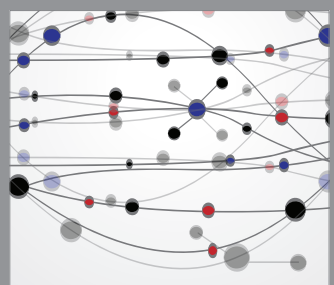

The Scientific World Journal
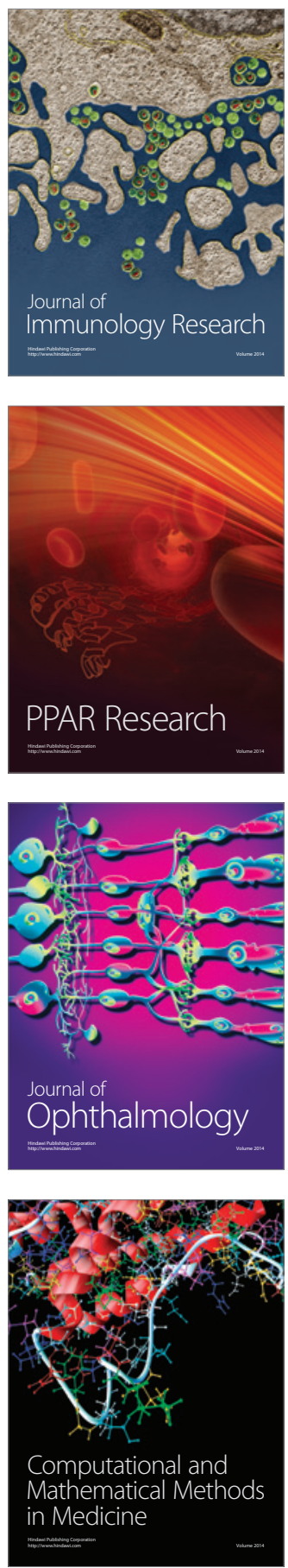

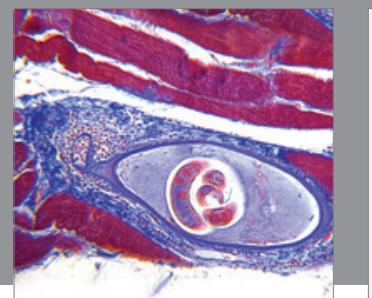

Gastroenterology

Research and Practice
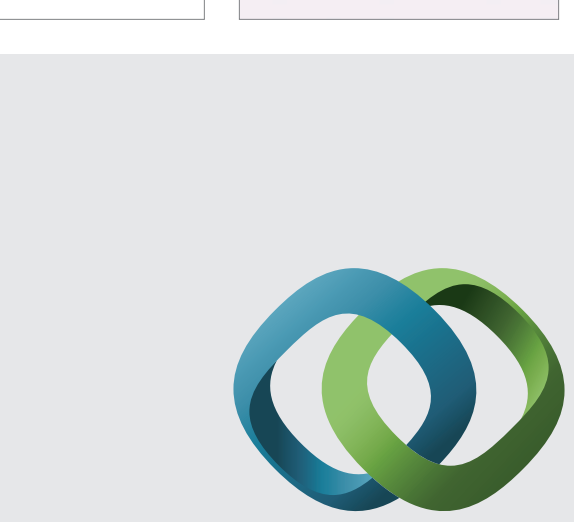

\section{Hindawi}

Submit your manuscripts at

http://www.hindawi.com
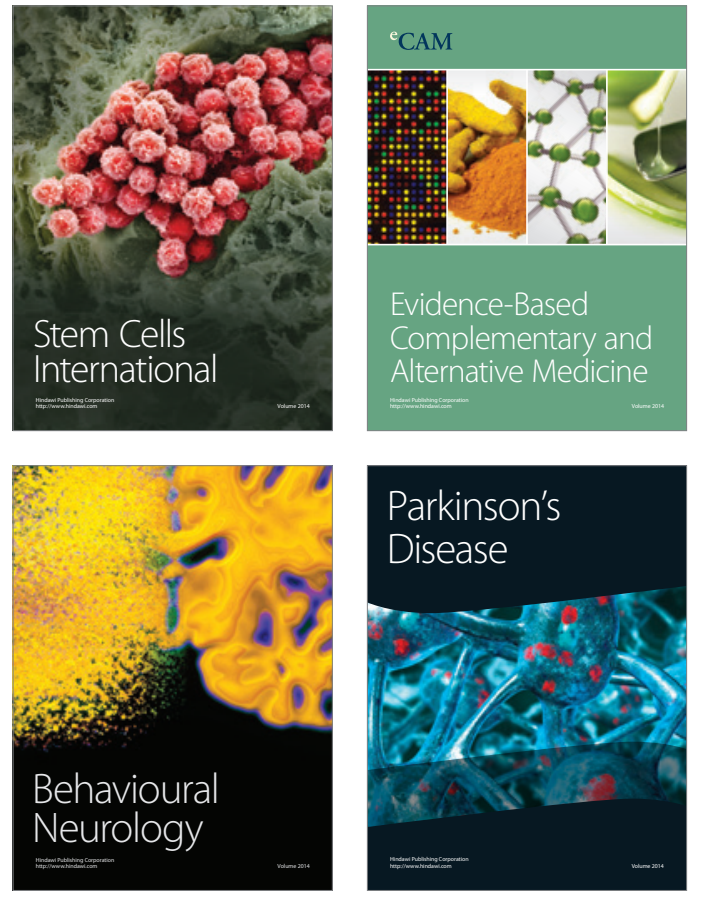
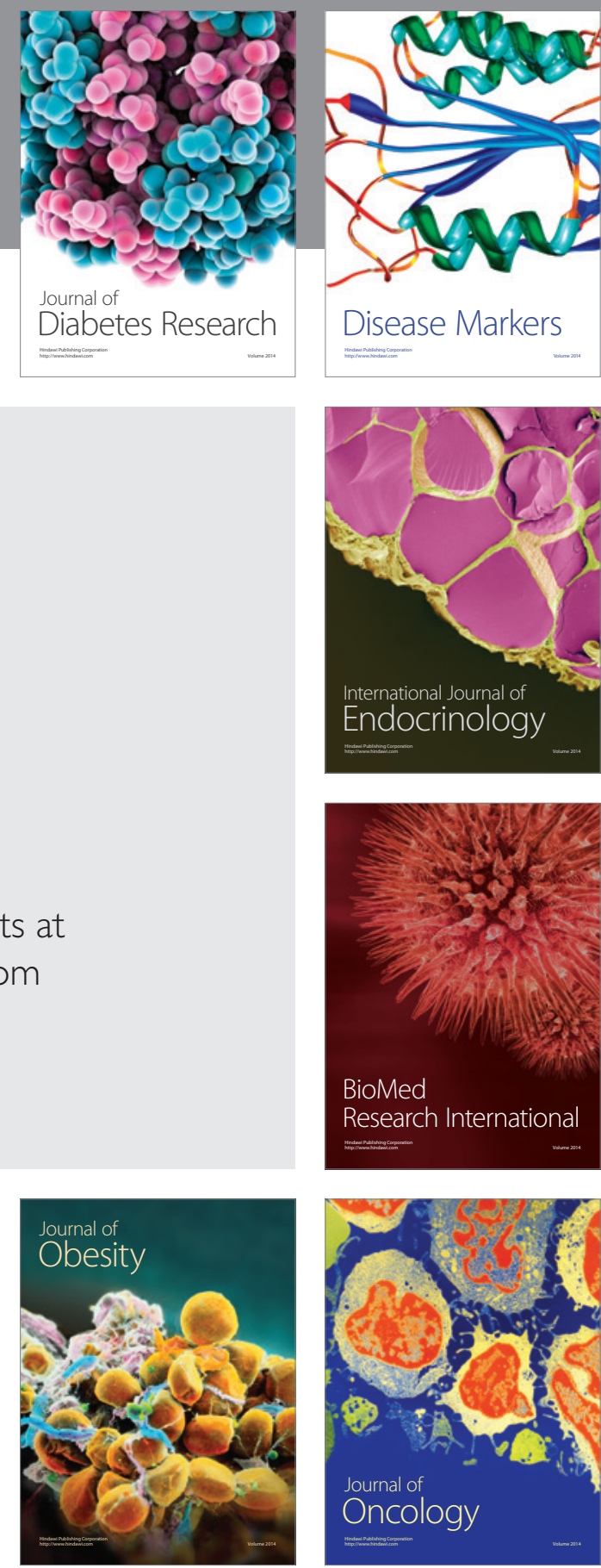

Disease Markers
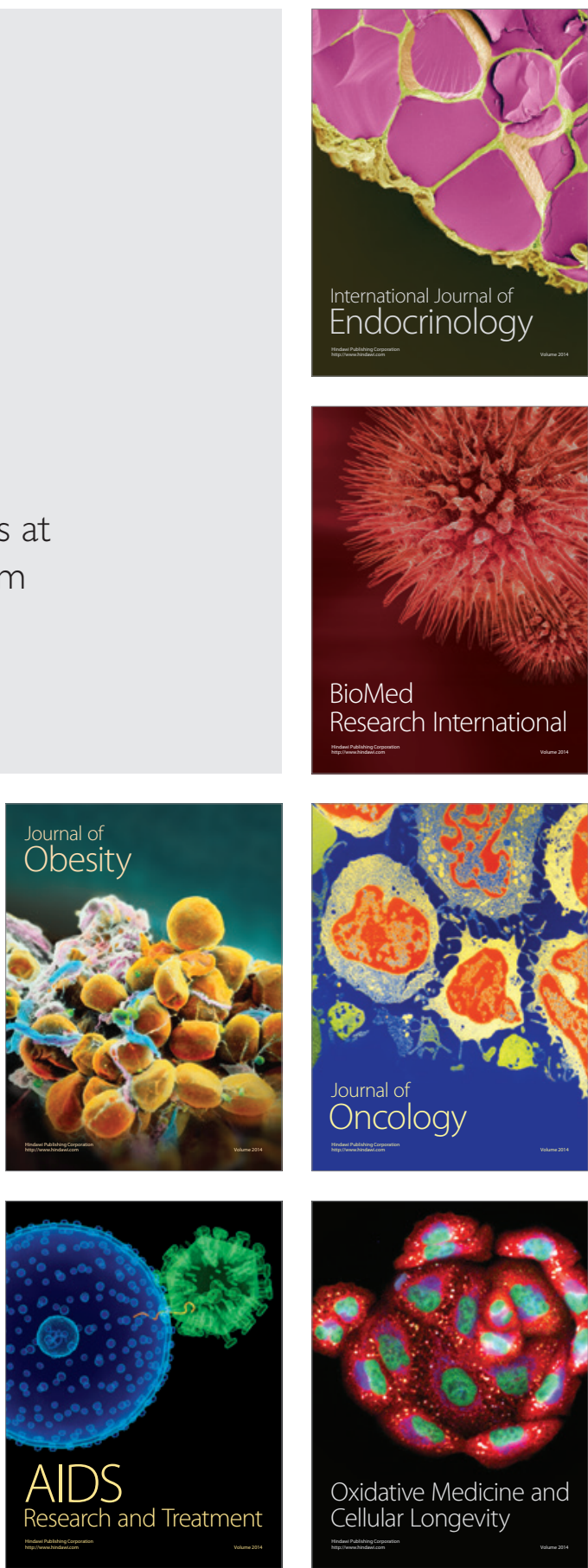